\title{
Model of Interaction Among Embedded Fuzzy Agents in Ubiquitous Computing Environments
}

\author{
Ricardo Rosales, Donald Rodriguez, Dora-Luz Flores, Luis Palafox, Manuel Castanon-Puga, \\ Carelia Gaxiola-Pacheco \\ Faculty of Chemical Sciences and Engineering, Autonomous University of Baja California, \\ Tijuana, Mexico
}

\begin{abstract}
This research is motivated by the need to propose a model for studying the people interaction in educational spaces such as interactive museums, assisted by ubiquitous computing environments and aided of fuzzy logic. People are represented as mobile agent software, which has certain characteristics, limited knowledge and the necessity to interact with others to achieve individual or collective goals in a specific or dynamic context. Fuzzy agents have the capacity to deal with the uncertainty and imprecision presents by user's interaction. We propose a model of interaction context-dependent among embedded fuzzy agents in ubiquitous computing environments that facilitate the interaction between people and the interactive museum, taking into account its ontology, autonomy, proactivity, mobility and social skills.
\end{abstract}

\section{Introduction}

The evolution of computational science to date is characterized by five trends: ubiquity, interconnection, intelligence, human-oriented and delegation[1]. The significance of two of these trends intelligence and delegation involves building computer systems that are able to act in place of the people, i.e. the possibility of first act independently and secondly, the ability to represent their interests in the best possible way when interacting with other people or systems.

The different ways that the user can interact with educative spaces (i.e. interactive museum) making a need how the applications can adapt (even automatically) to the situations, improving interaction[2]. The computation presence in ubiquitous way gives to the users the expectation that it is possible to access information and services anywhere. Besides that, the mobility provided by the ubiquity makes the user context, like the location people and objects around, become more dynamic to interact. A way to improve the support to the humancomputer interaction is to improve the communication during the interaction, making the computer able to process the user contextual information, devices and the system communication, allowing the implementation of more useful computational systems (context-awareness)[3].

In ubiquitous computing environments, computing is embedded in different devices, mobile terminals ,networks of sensors even machines with which we interact with various purposes in our daily lives (vending machines, , cars, , televisions, computers) and most of them have wireless access links.

In addition, there is a limited availability of computing resources (application and network systems), so these systems require the ability to adapt to it, so they can operate efficiently [4].

A key feature of ubiquitous computing systems is the ability to adapt their behaviour based on user activity and context[5]. The objects of the environment, have computing and communication capabilities, which makes a network intelligent devices and sensors in an environment that acts as a global interface between users and information systems, promoting communication between objects and allowing sensitivity to context, which helps reduce the configuration effort on the part of users. A key issue in the design of such adaptive systems is the reconfiguration that facilitates communication with users by building integrated applications dynamically[6].

\section{Methodology}

We use the meaning of sending the computer to a "background", refers to two different but related concepts, the first one is the literal meaning, that computer technology should be integrated into objects, things, tasks and daily environments. And the second one is that integration must be done, so that the introduction of computers into these things or objects do not interfere with the activities for which they are interact and always provide a convenient, simple and useful objects with properties such as communication, memory, context-sensitive and reactive. The incorporation of autonomous capabilities can significantly increase its power, especially in highly dynamic environments and multiple agents[7].

Figure 1 depicts our adaptive system separated in three agents: user, domain and adapter. This adaptive 
system determines the actual adaptation of the user's interaction. In order to identify a user's learning need, the Adapter agent applies rules for comparing the situational requirements with properties of the user. This means, both the Domain agent and the Adapter model serve as input-parameters for identifying a user's interaction need. The Domain agent defines the target (knowledge) state, and the Adapter model depicts the actual knowledge or competence state of a user. Additionally, relations from the Domain model (e.g., a prerequisite relation) can be part of the rules of the Adapter agent.

While the roles of the User and Adapter agents are obvious, the importance of the agent domain and its impact on the adaptation are often underestimated. We need to have four main functions of the Domain agent.

First, the domain agent forms the basis of all the inferences and predictions that can be made from the user's interaction with the system. Second, the system can only change aspects of the application, which are described in the domain agent. Third, in most cases it forms the basis of the adapter agent. Because the adapter model usually is realized as overlay of the domain agent, the domain agent determines the adapter agent scope. Fourth, the domain agent holds the measurable characteristics (e.g., skills) that are needed for evaluating the system's effectiveness.

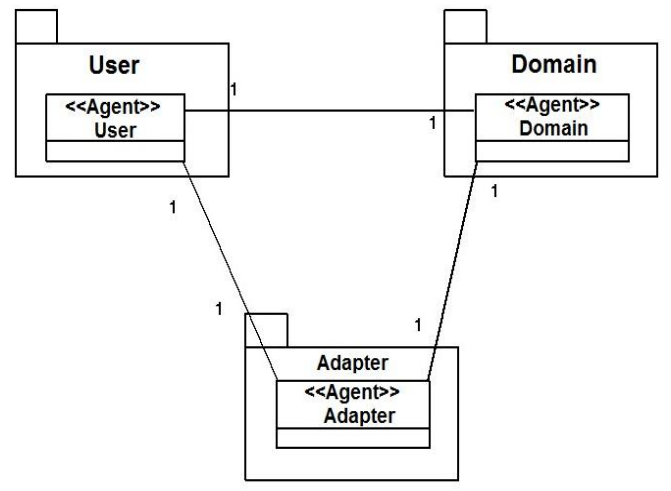

Figure 1. Main elements of the Adaptive System

In the Figure 2 schematically shows the involved three elements of the adaptation process and their interrelations in a) Adapter Agent is directly related to the agent process which is responsible for processing all applications for the users, processes all content and services according of user preferences, the results of this relationship of Processes Adapter which offers to the users all adapted processes based on user profile, offering contents or services required. b) Domain Agent is directly related to the resource agent which is responsible for managing all the resources and content that the user needs as a result of their interactions in the adaptive system, has the ability to offer multiple resources, the result of this relationship it's the domain resource which allows resources to deliver content according to the user profile. c) User Agent is directly related to the agent profile allowing the user to identify and relate directly to their profile, the profile agent manages and controls all user profiles that have interacted in the adaptive system, the resulting of this relationship is the user profile which allows the user to fully identify and to know the history and preferences.

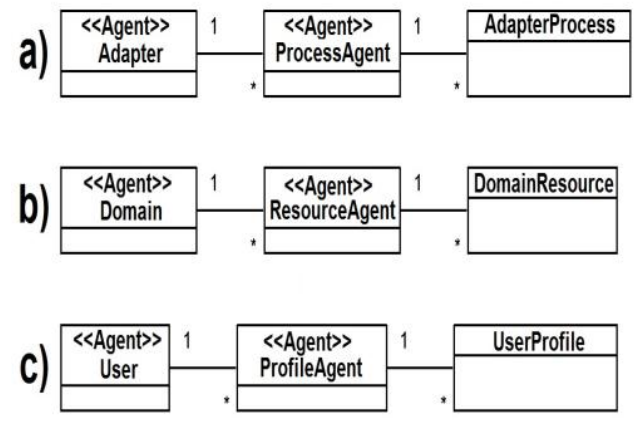

Figure 2. Relation process of the: a) Adapter Agent b) Domain Agent and c) User Agent

Although adaptive systems provide a mechanism for easing implementation complexity at the implementation level, the complexity inherent in the problems provide a solution remains a challenge.

We help us further applying a simplified AgentOriented Software Engineering (AOSE), methodology, in this is captured in two main model views: an organizational view which specifies the types of agents and roles, and an operational view which specifies the interaction constrained by goals and plans of actions to achieve those goals.

The agents are usually organized in a network, where an agent interacts only with its immediate neighbours in the network. Self-organization is defined as "the mechanism or process that allows the system to change its organization without explicit external command during runtime" [8]. This can be used in networks of agents to improve behaviour, interaction and cooperation.

\section{Fuzzy Context-Aware Environment}

We are modelling an user-friendly environment, efficient and distributed services, to support human interaction; the users are surrounded and helped for a digital context-aware environment based on a model of interaction. These aspects refer it to operations associated to contextual sensing, contextual adaptation and contextual resources, controlling the presentation of information and services to the user, automatic execution of environment actions. The Figure 3 shows such an adaptive system model, it is composed by three different components: the user model, adapter model, and domain model. The adapter model that will run all the services, 
interactions and contents, allow the awareness of context in different situations. Therefore agent adapter will be implemented; one for each entity is able to collaborate, information interchange via and services with others programs to solve complexes problems [9].The other component is the user model, will trigger the interactions and the time of its occurrence, have an agent user that contains the information about the user like preferences, layout, context, communication and interaction type.

The domain model has all the objects and contents that compose the scene to interact with the users. We have the domain agent that contains description of the content, interaction timeline and interaction media. In this model we use data of the user to offers content and services according with user's profiles.

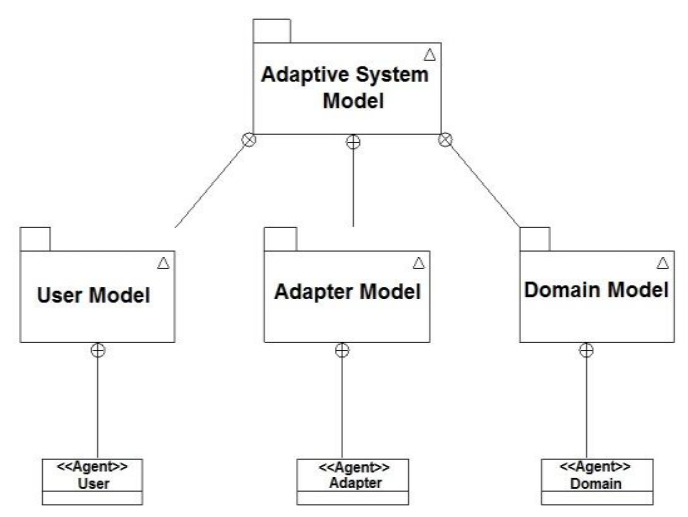

Figure 3. Adaptive System Model

The figure 4 it's a scheme to represent the interactions between entities in a context-aware environment. In the scheme, is possible to observe the existence of well-defined interactions, this environment is the means where the agent gets information and can act. The user-network interaction represents the interactions between one user (or multi-user) and the native network applications. The applications-network interactions cover the relationship between all applications (agents, programs, etc.) and the network. The userapplications interactions represent the interactions between user, via several kinds of interfaces and all applications of the environment. The completeinteractions well-defined scope of full interactions that happen involving user, network and applications.

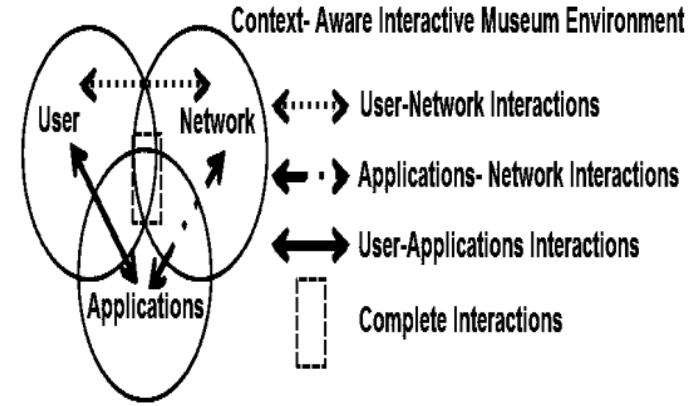

Figure 4. Interactions Scheme in a Context-Aware Interactive Environment

In this research we will propose use fuzzy logic to help MAS to represent more accurately the behaviour of interactive people's activities; where agents decide according to fuzzy-logic rules. This multi-agent system consists mainly of two types of agents: mobile agents who travels and interact just like the individuals moves along space and static agents who are mainly located on the buildings and facilities, which goal is to aid the mobile agents by providing them with the relevant information (site description, location, customizable services, etc.) so they can make decisions and take actions about their interaction. Arbitrarily many mobile agents (representing people who can have or not some grade abilities) of different kinds can exist in any space. In this context, the agents have needs and different levels of ability that can be endowed with various rules and strategies among select actions to meet their needs. Each mobile agent has some properties that influence his behaviour: grade of interaction and type of interaction. The properties are best described using type-2 fuzzy membership functions [10]. It's very hard to measure and classify accurately (without uncertainty) the grade and kind of interaction of any individual, and the classification process turns more complicated because all people exhibits different kind of behaviours, each one in a different grade, at the same time.

However, the main cause of uncertainty is humans occupying these environments as their behaviours and interactions are dynamic and non-deterministic, different words mean different things to different people[11].

\section{Case Study}

As a case study carried out the observing and modelling scenes on educative spaces (interactive museums) that may represent a real example, because they have a variety of interactive exhibitions and shows various situations that can rise due to the presence of groups of people. In the first instance due to the extent of its facilities and the dynamic that presents product of the daily activities, the installations of the Trompo Museum, located in 
Tijuana, Mexico is a magnificent place for the case study because is an interactive educative museum dedicated to children and teenagers and its primary goal is to be a place where they can interact and play while they are learning. We analysing the behaviour of users under 6 to 15 years, in some cases they are influenced and limited by their own schools guide, museum guides, affecting interactive motion and limiting the emergent behaviour, based on rules imposed, affecting innate learning of the individual.

The type of behaviour and learning when it is not influenced by guides it's so different, its innate and natural, the children have more autonomy looking for ways with an innate behaviour for interact with the exhibition modules, are not pressed in time, in their trajectories, impacting learning in a way through the interaction and play.

We analysing behaviour patterns of interactive museum environment, such as where they go, where they interacting, with whom are accompanied, interaction time, label reading time, fatigue, family and social behaviour, return visits, preference's services, exhibit information impact, visitor's attitudes, behaviours, misconceptions and interests. This information is crucial to building models to help in the design presentation format, signposting, labels, objects, layout, media, information, ability attention to follow directions, time spent, attitudes and other reactions. This improve the measurement and evaluation methods for assessing visitor learning, short and long-term impact of exhibit experiences, involvement, effort, social behaviour patterns, attendance and post visit interests. Nowadays we are in the process of collecting and analysing information to get feedback to the novel adaptive system modelling to ensure high quality of interactive services. This model consists in six types of agents and has interaction between them. The Museum Manager agent is the most important agent of the model, these centralizes all the user requests. This agent manages all the content of the museum, can find and adapt the service according to user profile, avoid request overloading. It also keeps the knowledge base using learning techniques.

The adapter agent is to analyse all the user requests, helps users to request services. It uses fuzzy logic algorithms and reasoning, to decide which service or proposed content is the best among the various proposals received. The agent user running on the user device, it's the key to communicate with other agents involved to receive or give data according to their user's profile. User Agent Museum provides to the user agent specialized processing information directly related to the museum, serves as an interface between the user and the other agents involved to receive more information according to user profile

The domain agent contains knowledge to the physical environment, information such as location, content of the museum, security services to data access also contains all the logical information of the museum's exhibitions and all learning content.

The domain museum provides to the domain agent, specialized information related to the content of the museum, serves as an interface between the domain and the other agents involved to provide information and content according to user profile

In the Figure 5 explains in detail the relationships of the agents making up the context-aware environment of the interactive museum

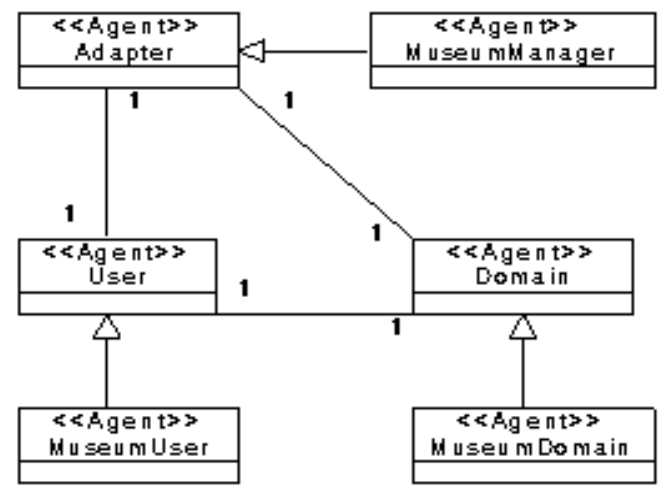

Figure 5. Interactions Scheme in a Context-Aware Interactive Museum

\section{Conclusion}

One of the reasons that motivate the use of agents is because the intelligent agents are seen as entities that emulate or simulate mental processes rational behaviour, like personal assistants, where agents are entities that help users accomplish a task such as finding ways to improve the interaction through an interactive museum. The intelligent agents represent systems that interact with their environment cognitively. They specialize in the pattern of perceiving, processing and knowledge representation to act accordingly. The use of MAS permits support personalized interaction, intelligent and active environment. That represents an approach to enhance adaptive systems with applications helps of Fuzzy Logic that can adapt and configure itself according to user's activities and user's profile.

We need identify what type of support is required for creating context-aware applications and developing a set of tools for rapid prototyping of context-aware applications. Such is the development of context sensitive providers that can help in sensing various types of contexts and allow access to other agents, taking into each device account that the infrastructure can provide different kinds of contexts. These may use different reasoning or learning mechanisms such as fuzzy logic. 
The most common context-sensitive applications not provide any generic mechanism for writing rules about contexts, inferring high-level contexts and the organization of the range of possible contexts. The problem of the contexts in standalone applications, distributed heterogeneous, where the entity has its own notion of context in terms of their views. To interact with other entities, the entity must know the relationship between his view and perspective of others. Therefore, for this research the main interest is the behaviour of people who perceive, remember and decide interact in an educative spaces according to their profiles, knowledge and adaptations.

\section{Acknowledgements}

We would like to thank the many people who made this research possible as we as the Mexican National Council for Science and Technology (CONACYT), Autonomous University of Baja California and the Museum El Trompo for the economic support granted for this research.

\section{References}

[1] Wooldridge, M (2002). An Introduction to Multi-agent System, John Wiley \& Sons, New York.

[2] Schmidt, A. (2000) 'Implicit Human Computer Interaction Through Context', Personal Technologies of Springer (4), pp. 191-199.

[3] Dey, A. (2000) 'Towards a Better Understanding of Context and Context-Awareness'. In Proceedings Workshop on The What, Who, Where, When, and How of Context-Awareness, The Hague, Netherlands.

[4] Ito, T. Takahashi, T. Suganuma, T. and Shiratori, N. (2010) 'Design of Adaptive Communication Mechanism for Ubiquitous Multiagent Systems' of Journal Information Processing (18), pp. 175-189.

[5] Privat, P. (2002) 'Des objets communicants a la communication ambiante', Les Cahiers du Numerique 3(4):2344.

[6] Saif, U. Pham, H. Mazzola, J. Waterman, J. Terman, (2003) 'A case for goal-oriented programming semantics'. In Proceedings Workshop on System Support for Computing, Seattle, USA.

[7] Sarne, D. Barbara, J. (2007) 'Estimating Information Value in Collaborative Multi-Agent Planning Systems', In Proceedings Conference Autonomous Agents and Multiagent Systems, NY, USA.

[8] Serugendo, G. Gleizes, G. and Karageorgos, A. (2005) 'Self-organization in multi-agent systems', The Knowledge Engineering Review, 20(2), pp. 165-189.
[9] Moreira, D. Walczowski, L. (1997) 'Using Software Agents to Generate VLSI Layouts', of the Intelligent Systems and Their Applications in IEEE Expert, (12), pp. 26-32.

[10] Macal C. North, M., Rogers, A. Jennings, A. Stefanovitch, R. (2005) 'Agent-Based Modelling and Simulation', In Proceedings of Winter Simulation Conference, Orlando, USA.

[11] Mendel, I. John, R.(2002) 'Type-2 Fuzzy Sets Made Simple' Fuzzy Systems on IEEE, (10), pp. 117-127. 